\title{
WILEY-VCH
}

DOI: $10.1002 /$ ((please add manuscript number))

Article type: Full Paper

\section{Shunt-Blocking Layers for Semitransparent Perovskite Solar Cells}

Maximilian T. Hörantner ${ }^{\dagger}$, Pabitra K. Nayakł, Sabyasachi Mukhopadhyay, Konrad

Wojciechowski, Clara Beck, David McMeekin, Brett Kamino, Giles E. Eperon and Henry J. Snaith*

$\dagger \mathrm{M} . \mathrm{H}$ and PN have contributed equally to this work.

M. T. Hörantner, Dr. P. K. Nayak, K. Wojciechowski, C. Beck, David McMeekin, Dr. Giles E. Eperon, Prof. H. J. Snaith

Department of Physics, University of Oxford, Clarendon Laboratory, Parks Road, Oxford, OX1 3PU, UK

E-mail: Henry.Snaith@physics.ox.ac.uk

Dr. S. Mukhopadhyay

Department of Materials and Interfaces, Weizmann Institute of Science, Rehovot, 76100, Israel

B. Kamino

Oxford Photovoltaics, Begbroke Science Park, Woodstock Road, Oxford, OX5 1PF, UK

Keywords: perovskite, solar cell, semi-transparent, siloxane, shunt-blocking

Perovskite solar cells have shown phenomenal progress and have great potential to be manufactured as low-cost large area modules. However, perovskite films often suffer from pinholes and the resulting contact between hole- and electron transporting layers provides lower resistance (shunt) pathways, leading to decreased open-circuit voltage and fill factor. This problem is even more severe in large area cells and especially in the case of neutral colour semitransparent cells, where a large absorber-free area is required to provide the desired transparency. Herein, we present a simple, inexpensive and scalable wet chemical method to block these "shunting paths" via deposition of transparent, insulating molecular layers which preferentially bind to the uncovered surface of the electron collecting oxide, without hindering charge extraction from the perovskite to the charge collection layers. We show that this method improves the performance in semitransparent cells, where the 


\section{WILEY-VCH}

enhancement in open-circuit voltage is up to $30 \%$ without negatively impacting the photocurrent. Using this method, we achieved an efficiency of $6.1 \%$ for a neutral colour semitransparent perovskite cell with $38 \%$ average visible transmittance. This simple shunt blocking technique has applications in improving the yield as well as efficiency of large area perovskite solar cells and light emitting devices.

\section{Introduction}

Organic-inorganic perovskite materials have been proven to be an efficient solar cell photoactive material and devices with power conversion efficiencies (PCEs) exceeding 20\% for small area cells have been demonstrated ${ }^{[1]}$. The crystal formation of the perovskite films is a widely studied topic ${ }^{[2,3]}$, different processing techniques have been used to grow crystalline perovskite films ${ }^{[4-6]}$, which makes this material very versatile, also in terms of manufacturing. The thin-film coverage and the morphology have been shown to affect the performance of devices ${ }^{[7]}$. Single step, solution processed films are prepared from lowviscous ionic solutions where the crystal formation is often very rapid. As a result the films can produce a "dewet" morphology as they grow, leaving pinholes in the thin-films ${ }^{[8]}$.

Pinholes in the absorber thin-films cause a loss in the ability to absorb the full photon flux and therefore reduce the achievable photocurrent density. Moreover, these pinholes allow direct contact between electron (n-type) and hole (p-type) transporting layers. These contacts create shunt paths (reduced shunt resistance $\left(\mathrm{R}_{\mathrm{SH}}\right)$ ) in the solar cell architecture. Lower $\mathrm{R}_{\mathrm{SH}}$ in solar cells reduces the fill factor $(\mathrm{FF})$ and the open-circuit voltage $\left(\mathrm{V}_{\mathrm{OC}}\right)$ and thus the PCE of the cell ${ }^{[9]}$. Improving the coverage of perovskite films by various optimized annealing, solvent dripping or other post treatments typically inhibits the shunting ${ }^{[5,10-13]}$. Most of such techniques result in smaller perovskite crystallites, which cover the surface in an improved manner, although this may be detrimental for maximizing device performance; crystal boundaries are thought to be sites that can cause non-radiative recombination ${ }^{[14]}$. 


\section{WILEY-VCH}

Due to industrial demand for building integrated photovoltaics, a number of reports presenting semitransparent devices have been published ${ }^{[15-17]}$. One approach is to use a thinner $(\sim 80-200 \mathrm{~nm})$ perovskite layer below the absorption depth to enhance the transmission $^{[15,16,18-20]}$. Devices applying this technique have reached efficiencies of $7.8 \%$ at an average visible transmittance (AVT) of $37 \%{ }^{[18]}$. However, such solar cells do not provide colour neutrality, which limits their use in building integrated applications: Due to the nature of the semiconductor absorption with density of states increasing from the bandgap, a continuous thin film of methylammonium lead iodide perovskite (with bandgap $\sim 1.6 \mathrm{eV}$ ) will tend to look brown. However, this is not a desirable colour for building-integrated applications, there is most demand for neutral-coloured solar cell windows. An alternative technique to obtain neutral colour semitransparent perovskite solar cells has been developed by Eperon et al. through a perovskite dewetting technique ${ }^{[21],[22]}$. A thick perovskite film is processed in such a way as to create a microstructured array of perovskite 'islands'. Each island is thick enough to absorb the majority of light below the bandgap, so appears black, and is too small to be seen with the bare eye. The combination of fully absorbing islands and transparent regions results in neutral colour devices. Though the reduced coverage of the absorbing layer provides higher transmittance through the device, it has the disadvantage of allowing shunting paths between the perovskite islands. Consequently, such devices yield low $\mathrm{V}_{\mathrm{OC}}$ and $\mathrm{FF}{ }^{[21,22]}$ when compared to fully covered films ${ }^{[18,23]}$.

As such, a method to selectively fill the regions between the perovskite islands with an electrically insulating materials in such semitransparent devices should greatly increase their open-circuit voltage and fill factor, and thus overall efficiency. In addition, such a "shuntblocking" technique should be advantageous for opaque devices, filling in pinholes resulting from defects in fabrication to minimise the impact of such pinholes upon device performance. We have previously shown that employing an insulating metal oxide honeycomb architecture, 


\section{WILEY-VCH}

where perovskite material is embedded in the carefully designed pores of the scaffold, can reduce the shunting problem ${ }^{[17]}$. However, this technique requires multiple fabrication steps and has as of yet failed to deliver colour neutral transparency.

Alkyl silanes can make well covering molecular monolayers on different semiconductor and metal oxide surfaces, and it has been shown that a monolayer of long alkyl chain silane molecules can act as an electronic insulating barrier for charge transport ${ }^{[24]}$. Yaffe et al. demonstrated that the conductivity of alkyl based molecular monolayer attached to a silicon surface exponentially decreases with the molecular length ${ }^{[25]}$. Previous work has also shown that short alkyl silanes with functional groups used as effective interfacial layers in inorganicorganic hybrid solar cell ${ }^{[26]}$ and perovskite solar cells - to modify device performances ${ }^{[27]}$.

Here we present a simple wet-chemical technique to block shunting paths in partially covered perovskite films via the use of an insulating octadecyl-siloxane (OTS) molecular layer. We show that this layer preferentially attaches to the exposed areas of electron transporting $\mathrm{TiO}_{2}$, without obstructing the charge transport through the perovskite. As such, this treatment acts to selectively block shunt pathways and resultantly improves the solar cell performance, leading to increased open-circuit voltage and a PCE of $6.1 \%$ for neutral colored semitransparent perovskite cells with a full device AVT close to $40 \%$.

\section{Results and Discussion}

We employed an inexpensive and commercially available molecule Trichloro(octadecyl)silane $\left(\mathrm{CH}_{3}(\mathrm{CH} 2)_{17} \mathrm{SiCl}_{3}\right.$; OTS) (Figure 1a) as a surface modifier by dissolving it in toluene and immersing the perovskite-coated substrates for 10 minutes with subsequent toluene rinsing and drying at $60^{\circ} \mathrm{C}$ (schematic shown in Figure 1b). In reaction conditions, OTS molecules hydrolyse to corresponding alkyltrihydroxy silanes (due to 


\section{WILEY-VCH}

presence of about $0.05 \%$ water in the toluene), then intermolecular condensation of -SiOH groups on neighbouring molecules form $\mathrm{Si}-\mathrm{O}-\mathrm{Si}$ (siloxane) bonds ${ }^{[28,29]}$. The hydroxy groups on $\mathrm{TiO}_{2}$ surfaces act as anchoring points for the alkylsiloxane or alkyltrihydroxy silanes by formation of Ti-O-Si bond ${ }^{[30],[31]}$. Due to the condensation reaction between silane molecules and $\mathrm{TiO}_{2}$ surface, the alkyl chains are expected to be packed as we illustrate in Figure 1c. It has been previously reported that assembly of OTS on a $\mathrm{TiO}_{2}$ surface results in a density of 4.3-4.8 molecules $/ \mathrm{nm}^{2}{ }^{[32]}$, and on $\mathrm{SiO}_{2}$ this results in a surface which has a water contact angle of 112-120 degrees ${ }^{[33]}$. To confirm that our treatment results in a similar coverage on compact $\mathrm{TiO}_{2}$, we measured the water contact angle of the $\mathrm{TiO}_{2}$ films with the OTS treatment, and found a value of 112 degrees (Figure S1), in good agreement with previous reports of well-packed monolayers.

In order to find out whether the insulating alkyl silane binds selectively to the uncovered area between the perovskite islands, we used conducting atomic force microscopy (c-AFM) on the pre and post silane-modified perovskite films on $\mathrm{TiO}_{2}$ as well as modified $\mathrm{TiO}_{2}$ surface after removing the perovskite layer. Figure 1d shows the topography image of partially covered perovskite layer; we show the current mapping of the same area in Figure 1e. We see that the current flowing through uncovered area is much higher than that on perovskite islands. In full devices these uncovered areas can act as shunting paths when in contact with hole transporting material. Figure $\mathbf{S 2}$ shows the c-AFM image of modified perovskite films. The normal topography image reveals that the incubation has not done any detectable modification on the film morphology, on the other hand the c-AFM image shows that the conductivity on the perovskite island area are generally higher or equal to the uncovered area. Figure 1f and 1g show the topography and current mapping of silane-modified surface, after the removal of perovskite with DMF. Even though the topography image does not show any features, the current mapping shows that the area initially covered with perovskite shows higher current density than the remaining area. That suggests that the silane molecules bind to 


\section{WILEY-VCH}

the exposed $\mathrm{TiO}_{2}$ surface. The FTIR spectrum (see Figure S3) of the silane modified surface (after removal of perovskite layer) shows IR features of alkyl chains.

Although we do not expect the silane molecules to rapidly attach to the perovskite film itself, there is a possibility of this happening, which could have the negative impact of inhibiting hole transfer from the perovskite to the hole-conductor. In order to asses if there is any negative influence on hole transfer, we performed a photoluminescence (PL) decay study. Continuous perovskite films on glass slides were treated with OTS solution and then coated with the hole conductor, 2,2',7,7'-Tetrakis[N,N-di(4-methoxyphenyl)amino]-9,9'spirobifluorene (spiro-OMeTAD). In Figure $\mathbf{S 4}$ we show the PL decay for the perovskite films with and without OTS treatment, and with and without coating with spiro-OMeTAD. PL decay curves shows that the quenching of PL due to hole transfer (manifested as sharper decay curves) is same for both OTS treated and untreated films indicating no negative effect of OTS treatment. We did not find evidence that the siloxane treatment changes the electronic transport properties at the perovskite and hole transporter interface, as reported by Zhang et al. ${ }^{[34]}$.

Furthermore, we compared the change in surface energy on $\mathrm{TiO}_{2}$ and perovskite islands through the attachment of the OTS. Thus we performed Chlorobenzene contact angle measurements on uncovered $\mathrm{FTO} / \mathrm{c}-\mathrm{TiO}_{2}$ substrates before and after the OTS treatment and compared them to substrates that were additionally covered with dewet perovskite films. We present the values and images in Figure S5 and notice an increase in contact angle on OTS treated $\mathrm{TiO}_{2}$ surfaces and a significantly lower increase on OTS treated partially covered perovskite films. These findings are consistent with the assumption that the OTS molecules preferentially attach to the uncovered $\mathrm{TiO}_{2}$ surfaces.

To gauge the effect of shunt blocking with the OTS treatment, we prepared solar cells with 2,2' $, 7,7^{\prime}$-Tetrakis[N,N-di(4-methoxyphenyl)amino]-9,9'-spirobifluorene (spiro-OMeTAD) as hole transporting material and $\mathrm{Au}$ as top electrode. Island-structured semitransparent devices 


\section{WILEY-VCH}

are prepared as described by Eperon et al ${ }^{[21]}$. In order to observe the effect of the treatment on device reproducibly as well as performance we present device statistics from a batch of 67 devices in Figure 2a-2d. We find that the standard deviation of working devices is much higher for the treated films than for the untreated ones (Figure 2a and 2c). On each substrate we tested 8 different pixels (area $0.09 \mathrm{~cm}^{2}$ ). We filter out the data points that show very low power conversion efficiency $(\mathrm{PCE}<0.1 \%)$. Although we note that several factors other than pinholes can be responsible for lower performance, we can observe that the general yield of working solar cells was improved by $5 \%$ upon treatment. Figure $\mathbf{2 a}$ and $\mathbf{2 b}$ show the histograms of the $\mathrm{V}_{\mathrm{OC}}$ and PCE of all working solar cells. We would like to point out that we not only enhanced open-circuit voltage and overall power conversion efficiency, but also largely improved the reproducibility of the devices, which is evidenced by significantly lower standard deviation of the treated samples. The $V_{O C}$ values of the semitransparent films reach $\sim 1 \mathrm{~V}$ that could so far only be achieved with fully covered perovskite films ${ }^{[10,21]}$.

Figure 2e shows the average JV characteristics of all functioning cells and the single JV curves for the best performing treated and untreated devices under illumination. The low coverage of perovskite leads to semitransparent devices with average visible transmittance (AVT) of ca. 45\% (see SEM image in Figure S6) without top electrode. The $\mathrm{J}_{\mathrm{SC}}$ of these semitransparent films correlates with the coverage and therefore results in a trade-off between efficiency and transparency in the devices ${ }^{[21]}$. Consequently, at a given AVT of the device the $\mathrm{J}_{\mathrm{SC}}$ will not be improved, as can be seen in the similarity of maximum currents of the different devices in Figure 2e. However, upon OTS treatment, we observe that the $\mathrm{V}_{\mathrm{OC}}$ and FF are significantly improved to reach a maximum PCE of $5.9 \%$ (average PCE of $4.6 \pm 0.8 \%$ ) compared to $4.3 \%$ in control cells (average PCE of $3.2 \pm 1.4 \%$ ). We show all the characteristic parameters in Table 1. We note that these cells show current-voltage hysteresis with and without the OTS treatment (Figure S7), consistent with previously reported results ${ }^{[22]}$, and the steady-state efficiency measured at the maximum power point is lower than the PCE derived 


\section{WILEY-VCH}

from the JV scan (Figure S8). The fact that the OTS treated devices function as solar cells and produce similar photo-current density suggests that the OTS molecules are not preventing the charge collection. The attachment of the long alkyl chains on the perovskite surface would have hindered the contact between perovskite and hole transporting material and extraction of photo-generated holes, in turn lowering the photovoltaic performance of those devices. Furthermore, as we propose that the interface between the perovskite domains and the charge collection layers is not affected, we assume that hysteresis or stabilized maximum power performance will remain unimproved upon treatment.

We fitted the averaged JV curves of the treated and untreated devices to a single-diode equivalent circuit model after Unger et al. ${ }^{[35]}$ to determine the series $\left(\mathrm{R}_{\mathrm{S}}\right)$ and shunting resistances $\left(\mathrm{R}_{\mathrm{SH}}\right)$ (see Figure S9). We find that $\mathrm{R}_{\mathrm{S}}=42.0 \mathrm{Ohm}, \mathrm{R}_{\mathrm{SH}}=1.29 \times 10^{3} \mathrm{Ohm}$ for the untreated and $\mathrm{R}_{\mathrm{S}}=119.9 \mathrm{Ohm}, \mathrm{R}_{\mathrm{SH}}=4.96 \times 10^{9} \mathrm{Ohm}$ for the post-treated cells, revealing a large increase in $\mathrm{R}_{\mathrm{SH}}$, which can be attributed to the insulating character of long alkyl chains blocking the contact between electron and hole transporting materials. The slight increase in series resistance could be speculatively caused by thickness difference in hole-transporting layer, which could stem from a change of the spiro-OMeTAD precursor solution wetting upon treatment ${ }^{[36]}$. We note that the real equivalent circuit of our semitransparent perovskite cells is a double heterojunction diode (compact $\mathrm{TiO}_{2} /$ perovskite/spiro-OMeTAD) in parallel with an n-p heterojunction comprising compact $\mathrm{TiO}_{2} /$ spiro-OMeTAD (see Figure $\mathbf{S 1 0}$ right). Hence, the shunt resistance, represented by the $\mathrm{TiO}_{2} /$ spiro-OMeTAD junction, is in fact a diode itself and the current which passes through it will have a bias dependence. Therefore, the one diode model, which assumes a fixed bias independent shunt resistance, only gives a first approximation to the changes which occur within the actual cell upon OTS treatment. We measured the JV-characteristic curves of $\mathrm{TiO}_{2} /$ spiro-OMeTAD and $\mathrm{TiO}_{2} / \mathrm{OTS} /$ spiroOMeTAD n-p diodes, prepared in the same way as solar cell devices but without the perovskite layer, and find that the blocking behaviour of the OTS treated diode is extended to 


\section{WILEY-VCH}

much higher voltages when compared to the untreated diode (Figure S10 left). By combining these measurements with calculated fractional JV data from a fully covered well performing device, ${ }^{[13]}$ where we replicate the significant gain in VOC upon OTS treatment.

Beyond the results we have presented above, we have further improved the device efficiencies: We either enhance the FF with a treatment of the perovskite films with iodopenta-fluorobenzene (IPFB), which we have previously shown to reduce the recombination at the interface with the hole-transporting material. ${ }^{[37]}$ Or we haveimproved the $\mathrm{J}_{\mathrm{SC}}$ and $\mathrm{FF}$ with a $\mathrm{TiCl}_{4}$ treatment of the $\mathrm{c}-\mathrm{TiO}_{2}$ layer, prior to perovskite deposition ${ }^{[38]}$. We show the JV curves of the resulting champion cells, which exhibit a PCE of $6.6 \%$ and 7.4\% for the IPFB and $\mathrm{TiCl}_{4}$ enhanced cells respectively in Figure S11.

Large area cells that are required for commercial applications could be more sensitive to the effect of shunting. We tested another set of devices with more than 7 times larger area $(0.71$ $\mathrm{cm}^{2}$ as we display in Figure S12) where the shunting effects are more prominent. We present the results in Figure S13, where we observe that the PCE is improved from $4.2 \%$ to $5.2 \%$ comparing cells without and with the OTS treatment. Again, the reason for this enhancement stems from the significantly improved $\mathrm{V}_{\mathrm{OC}}$ from $0.69 \mathrm{~V}$ to $0.89 \mathrm{~V}$.

The real world application of semitransparent perovskite solar cells requires both the top and bottom electrodes to be semitransparent, i.e. in our case the reflecting top Au electrode needs to be replaced. We fabricated a set of semitransparent devices using a laminated Nickel micro-grid (with an AVT of $85 \%$ ) as a top electrode, as reported previously ${ }^{[39]}$. We observe very little loss in performance, as compared to the devices incorporating a metal electrode, and achieve a record PCE of 6.1\%, which we present in Figure 3a. We note that in previous reports of employing this gird electrode, especially in semitransparent perovskite films, the PCE has typically been lower than the control devices, coming mostly from a slight drop in $\mathrm{V}_{\mathrm{OC}}{ }^{[22,39]}$. This may be due to the PEDOT employed being especially effective at penetrating pinholes through the perovskite and spiro-OMeTAD layer, which here are blocked by the 


\section{WILEY-VCH}

presence of the OTS layer. We used the measured total transmittance of the device, which we show in Figure $\mathbf{3 b}$, to calculate the AVT, which is high at $38 \%$. To the best of our knowledge, these resulting performance parameters currently represent the highest efficiency for a neutral colour semitransparent perovskite solar cell ${ }^{[15,20]}$. Thin-film perovskite devices, with brown-tinted colour, have been reported to be more efficient, with AVT of $\sim 37 \%$ and PCE of 7.8\%, but the presented surface-treated solar cells remain neutral coloured (Figure 3b) and are therefore very attractive for building applications. The best performing previous neutral-coloured semitransparent device had an AVT of $\sim 30 \%$ and PCE of $\sim 5 \%$.

\section{Conclusion}

In summary, we have presented a simple wet chemical technique where a molecular posttreatment of perovskite films on $\mathrm{TiO}_{2}$ substrates prevents the formation of shunting paths, which are detrimental for solar cell performance. In this treatment, alkyl-silaxone molecular layers preferentially bind to the $\mathrm{TiO}_{2}$ surfaces at pinhole sites without hindering charge transport across the active layers. In "island-structured" perovskite solar cells with a low surface coverage, we see a significant increase in the shunt resistance due to the post treatment, which results in a better solar cell performance and yield. In large area $\left(0.71 \mathrm{~cm}^{2}\right)$ cells, we observe an improvement in PCE by $20 \%$. We have achieved a remarkable $6.1 \%$ PCE for neutral colour semitransparent (entire cell AVT of 38\%) perovskite cells by using OTS assembly, which represents the highest efficiency/transparency combination reported todate. This low cost and easily scalable shunt path blocking technique will have wide application in improving the yield as well as efficiency of large area perovskite solar cell and light emitting devices, which is specifically advantageous for commercial exploitation of perovskite solar modules in BIPV applications.

\section{Experimental Section}




\section{WILEY-VCH}

Substrate preparation: The glass substrates were purchased from Pilkington Inc. with a coated FTO layer with sheet resistance of $15 \mathrm{Ohm} / \mathrm{sq}$. FTO was partially removed from the substrate via etching with zinc powder and $2 \mathrm{M} \mathrm{HCl}$ then cleaned sequentially with Hellmanex detergent, DI-Water, acetone and ethanol. Then $\mathrm{O}_{2}$ plasma treatment was done for 10 minutes. The $\mathrm{TiO}_{2}$ compact layer was deposited by spin-coating a solution of $0.71 \mathrm{~g}$ titanium isopropoxide and $0.07 \mathrm{~g} \mathrm{HCl}$ in $8 \mathrm{~mL}$ of ethanol, followed by sintering on a hot plate at $500{ }^{\circ} \mathrm{C}$ for $30 \mathrm{~min}$.

Perovskite film deposition and OTS treatment: Perovskite layers with low coverage were deposited after the technique developed by Eperon et al. ${ }^{[21]} .38 \mathrm{wt} \% \mathrm{CH}_{3} \mathrm{NH}_{3} \mathrm{I}$ and $22 \mathrm{wt} \%$ $\mathrm{PbCl}_{2}$ was dissolved subsequently in Dimethylsolfanoxide (DMSO) and stirred for 1 hour. $250 \mu \mathrm{L}$ of the solution was spin-coated on to a $3 \times 3 \mathrm{~cm}^{2}$ substrate with $2000 \mathrm{rpm}$ for 45 seconds and then annealed at $130{ }^{\circ} \mathrm{C}$ for 20 minutes. Spin-coating and annealing was done in air with $20 \%$ controlled humidity.

The substrates with partially covered perovskite layer were then immersed in toluene kept in a petri dish . Octadecyl-trichloro silane $(10 \mu \mathrm{l}$ per ml of toluene) was then added to the toluene solution. After 10 minutes of incubation at room temperature, the substrates were rinsed with toluene followed by drying at $60^{\circ} \mathrm{C}$ on a hotplate.

Device cell fabrication: For the fabrication of solar cell devices, the hole-transporting layer was deposited on top of the perovskite films by spin-coating a solution with $8.5 \mathrm{wt} \%$

\section{$2,2^{\prime} \quad, 7,7^{\prime} \quad$-Tetrakis[N,N-di(4-methoxyphenyl)amino]-9,9' -spirobifluorene (spiro-}

OMeTAD) in chlorobenzene and $30 \mathrm{~mol} \%$ lithium bis(trifluoromethane-sulfonyl)imide and $80 \mathrm{~mol} \%$ 4-tert-butylpyridine as additives, at $2000 \mathrm{rpm}$ for 60 seconds in the $20 \%$ humid air atmosphere. The $\mathrm{Au}(50 \mathrm{~nm})$ layer was evaporated on top of spiro-OMeTAD as the topelectrode. Transparent top electrodes were made with a flexible Nickel micro grid, as reported elsewhere ${ }^{[39]}$. 


\section{WILEY-VCH}

Solar cell testing: For measuring the performance of the solar cells, simulated AM 1.5

sunlight was generated with a class AAB ABET solar simulator. It was calibrated to give simulated AM 1.5, of $100 \mathrm{mWcm}^{-2}$ equivalent irradiance, using an NREL-calibrated KG5 filtered silicon reference cell. The mismatch factor was calculated to be 1.065 between $300-$ $900 \mathrm{~nm}$, which is beyond the operating range of both the KG5 filtered silicon reference cell and the perovskite test cells ${ }^{[40]}$. The current-voltage curves were recorded with a sourcemeter (Keithley 2400, USA). The solar cells were masked with a metal aperture defining the active area $\left(0.0929 \mathrm{~cm}^{2}\right.$ or $\left.0.71 \mathrm{~cm}^{2}\right)$ of the solar cells.

Optical and SEM characterization: The transmission measurements were carried out with an internally coupled integrated sphere in a Varian Cary 300 UV-visible spectrophotometer. The average visible transmittance was calculated by averaging over all total transmittance value within the visible spectrum $(400-780 \mathrm{~nm})$. Structural characterization was carried out with a Hitachi S-4300 field emission scanning electron microscope.

AFM measurements: The conductive AFM measurements were carried out with environment control SPM system (Solver P47, NT-MDT, Zelenograd, Russia) under constant $\mathrm{N}_{2}$ flow purge ( $<4 \%$ relative humility) in combination with Pt-coated Si probes (HQ:DPER-

XSC11/D; NanoAndMore GmbH).

\section{Supporting Information}

Supporting Information is available from the Wiley Online Library or from the author.

\section{Acknowledgements}

This work was supported by the EPSRC UK, the European commission through the MESO project and Oxford PV Ltd. SM acknowledges Department of Chemical Research Support, Weizmann Institute of Science and Council for Higher Education (Israel) for a PBC program postdoctoral research fellowship. We thank Sandeep Pathak for inspiring discussions.

Received: ((will be filled in by the editorial staff))

Revised: ((will be filled in by the editorial staff)) Published online: ((will be filled in by the editorial staff))

[1] M. A. Green, K. Emery, Y. Hishikawa, W. Warta, E. D. Dunlop, Prog. Photovoltaics 


\section{WILEY-VCH}

Res. Appl. 2015, 23, 1.

[2] G. E. Eperon, V. M. Burlakov, P. Docampo, A. Goriely, H. J. Snaith, Adv. Funct. Mater. 2013.

[3] M. Xiao, F. Huang, W. Huang, Y. Dkhissi, Y. Zhu, J. Etheridge, A. Gray-Weale, U. Bach, Y.-B. Cheng, L. Spiccia, Angew. Chemie 2014, 126, n/a.

[4] M. M. Lee, J. Teuscher, T. Miyasaka, T. N. Murakami, H. J. Snaith, Science 2012, 338, 643.

[5] J. Burschka, N. Pellet, S.-J. J. Moon, R. Humphry-Baker, P. Gao, M. K. Nazeeruddin, M. Grätzel, M. Gratzel, Nature 2013, 499, 3.

[6] M. Liu, M. B. Johnston, H. J. Snaith, Nature 2013, 501, 395.

[7] S. D. Stranks, P. K. Nayak, W. Zhang, T. Stergiopoulos, H. J. Snaith, Angew. Chemie Int. Ed. 2015, 54, n/a.

[8] M. D. McGehee, Nat. Mater. 2014, 13, 845.

[9] J. Nelson, The Physics of Solar Cells, Imperial College Press, 2003.

[10] G. E. Eperon, V. M. Burlakov, P. Docampo, A. Goriely, H. J. Snaith, Adv. Funct. Mater. 2014, 24, 151.

[11] M. Xiao, F. Huang, W. Huang, Y. Dkhissi, Y. Zhu, J. Etheridge, A. Gray-Weale, U. Bach, Y. B. Cheng, L. Spiccia, Angew. Chemie - Int. Ed. 2014, DOI 10.1002/anie.201405334.

[12] N. J. Jeon, J. H. Noh, Y. C. Kim, W. S. Yang, S. Ryu, S. Il Seok, Nat. Mater. $2014,1$.

[13] W. Zhang, M. Saliba, D. T. Moore, S. K. Pathak, M. T. Hörantner, T. Stergiopoulos, S. D. Stranks, G. E. Eperon, J. A. Alexander-Webber, A. Abate, A. Sadhanala, S. Yao, Y. Chen, R. H. Friend, L. A. Estroff, U. Wiesner, H. J. Snaith, Nat. Commun. 2015, 6, 6142.

[14] D. W. deQuilettes, S. M. Vorpahl, S. D. Stranks, H. Nagaoka, G. E. Eperon, M. E. Ziffer, H. J. Snaith, D. S. Ginger, Science (80-. ). 2015, 348, 683.

[15] E. Della Gaspera, Y. Peng, Q. Hou, L. Spiccia, U. Bach, J. J. Jasieniak, Y.-B. Cheng, 


\section{WILEY-VCH}

Nano Energy 2015, 13, 249.

[16] C. Roldán-Carmona, O. Malinkiewicz, R. Betancur, G. Longo, C. Momblona, F. Jaramillo, L. Camacho, H. J. Bolink, Energy Environ. Sci. 2014, 7, 2968.

[17] M. T. Hörantner, W. Zhang, M. Saliba, K. Wojciechowski, H. Snaith, Energy Environ. Sci. 2015, 8, 2041.

[18] J. W. Jung, C.-C. Chueh, A. K.-Y. Jen, Adv. Energy Mater. 2015, n/a.

[19] C.-Y. Chang, K.-T. Lee, W.-K. Huang, H.-Y. Siao, Y.-C. Chang, Chem. Mater. 2015, 27, 150702111029004.

[20] C. O. Ramírez Quiroz, I. Levchuk, C. Bronnbauer, M. Salvador, K. Forberich, T. Huemueller, Y. Hou, P. Schweizer, E. Spiecker, C. J. Brabec, J. Mater. Chem. A 2015, DOI 10.1039/C5TA08450D.

[21] G. E. Eperon, V. M. Burlakov, A. Goriely, H. J. Snaith, ACS Nano 2014, 8, 591.

[22] G. E. Eperon, D. Bryant, J. Troughton, S. D. Stranks, M. B. Johnston, T. Watson, D. A. Worsley, H. J. Snaith, J. Phys. Chem. Lett. 2015, 6, 129.

[23] C. Roldan-Carmona, O. Malinkiewicz, A. Soriano, G. Minguez Espallargas, A. Garcia, P. Reinecke, T. Kroyer, M. I. Dar, M. K. Nazeeruddin, H. J. Bolink, Energy Environ. Sci. 2014, 7, 994.

[24] S. A. DiBenedetto, A. Facchetti, M. A. Ratner, T. J. Marks, Adv. Mater. 2009, 21, 1407.

[25] O. Yaffe, Y. Qi, L. Scheres, S. R. Puniredd, L. Segev, T. Ely, H. Haick, H. Zuilhof, A. Vilan, L. Kronik, A. Kahn, D. Cahen, Phys. Rev. B 2012, 85, 045433.

[26] P. K. Nayak, L. Barnea-Nehoshtan, R. S. Kim, A. Shu, G. Man, A. Kahn, D. Lederman, Y. Feldman, D. Cahen, Energy Environ. Sci. 2013, 6, 3272.

[27] L. Zuo, Z. Gu, T. Ye, W. Fu, G. Wu, H. Li, H. Chen, J. Am. Chem. Soc. 2015, 137, 2674.

[28] A. N. Parikh, M. A. Schivley, E. Koo, K. Seshadri, D. Aurentz, K. Mueller, D. L. Allara, J. Am. Chem. Soc. 1997, 119, 3135. 


\section{WILEY-VCH}

[29] D. L. Allara, A. N. Parikh, F. Rondelez, Langmuir 1995, 11, 2357.

[30] N. Iguchi, C. Cady, R. C. Snoeberger, B. M. Hunter, E. M. Sproviero, C. a Schmuttenmaer, R. H. Crabtree, G. W. Brudvig, V. S. Batista, in Phys. Chem. Interfaces Nanomater. Vii, 2008, pp. C340-C340.

[31] J. Zhao, M. Milanova, M. M. C. G. Warmoeskerken, V. Dutschk, Colloids Surfaces A Physicochem. Eng. Asp. 2012, 413, 273.

[32] R. Helmy, A. Y. Fadeev, Langmuir 2002, 18, 8924.

[33] Y. Wang, M. Lieberman, Langmuir 2003, 19, 1159.

[34] J. Zhang, Z. Hu, L. Huang, G. Yue, J. Liu, X. Lu, Z. Hu, M. Shang, L. Han, Y. Zhu, Chem. Commun. (Camb). 2015, 51, 7047.

[35] E. L. Unger, E. T. Hoke, C. D. Bailie, W. H. Nguyen, A. R. Bowring, T. Heumuller, M. G. Christoforo, M. D. McGehee, Energy Environ. Sci. 2014, 7, 3690.

[36] N. Marinova, W. Tress, R. Humphry-Baker, M. I. Dar, V. Bojinov, S. M. Zakeeruddin, M. K. Nazeeruddin, M. Grätzel, ACS Nano 2015, 9, 4200.

[37] A. Abate, M. Saliba, D. J. Hollman, S. D. Stranks, K. Wojciechowski, R. Avolio, G. Grancini, A. Petrozza, H. J. Snaith, Nano Lett. 2014, 14, 3247.

[38] P. M. Sommeling, B. C. O’Regan, R. R. Haswell, H. J. P. Smit, N. J. Bakker, J. J. T. Smits, J. M. Kroon, J. A. M. van Roosmalen, J. Phys. Chem. B 2006, 110, 19191.

[39] D. Bryant, P. Greenwood, J. Troughton, M. Wijdekop, M. Carnie, M. Davies, K. Wojciechowski, H. J. Snaith, T. Watson, D. Worsley, Adv. Mater. 2014, 26, 7499.

[40] H. J. Snaith, Energy Environ. Sci. 2012, 5, 6513. 


\section{WILEY-VCH}
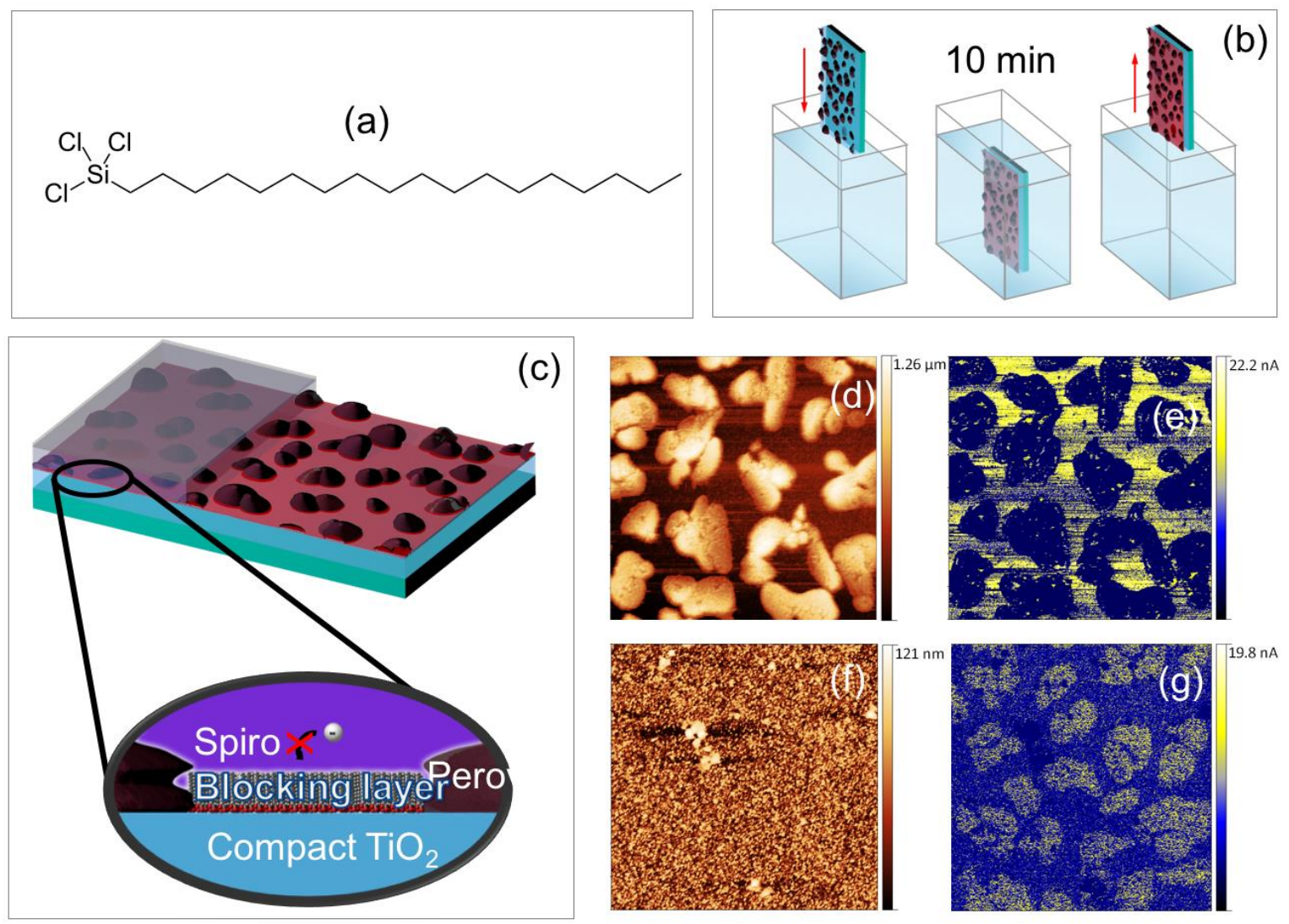

Figure 1. Representation of the $\mathrm{CH}_{3}\left(\mathrm{CH}_{2}\right)_{17} \mathrm{SiCl}_{3}$ chemical structure (a). Schematic of the dipcoating process for the selective assembly of the Silane blocking layer (b). Sketch of the components of the island-structured perovskite device with siloxane blocking layer between perovskite domains (c). Conventional (d) and conductive (e) AFM images of dewet perovskite domains on top of compact $\mathrm{TiO}_{2}$ layer without OTS treatment. Conventional (f) and conductive (g) AFM images of compact $\mathrm{TiO}_{2}$ layer after OTS treatment and subsequent removal of perovskite domains via DMF immersion. All AFM images represent a 40 x $40 \mu \mathrm{m}$ scanned area. 


\section{WILEY-VCH}
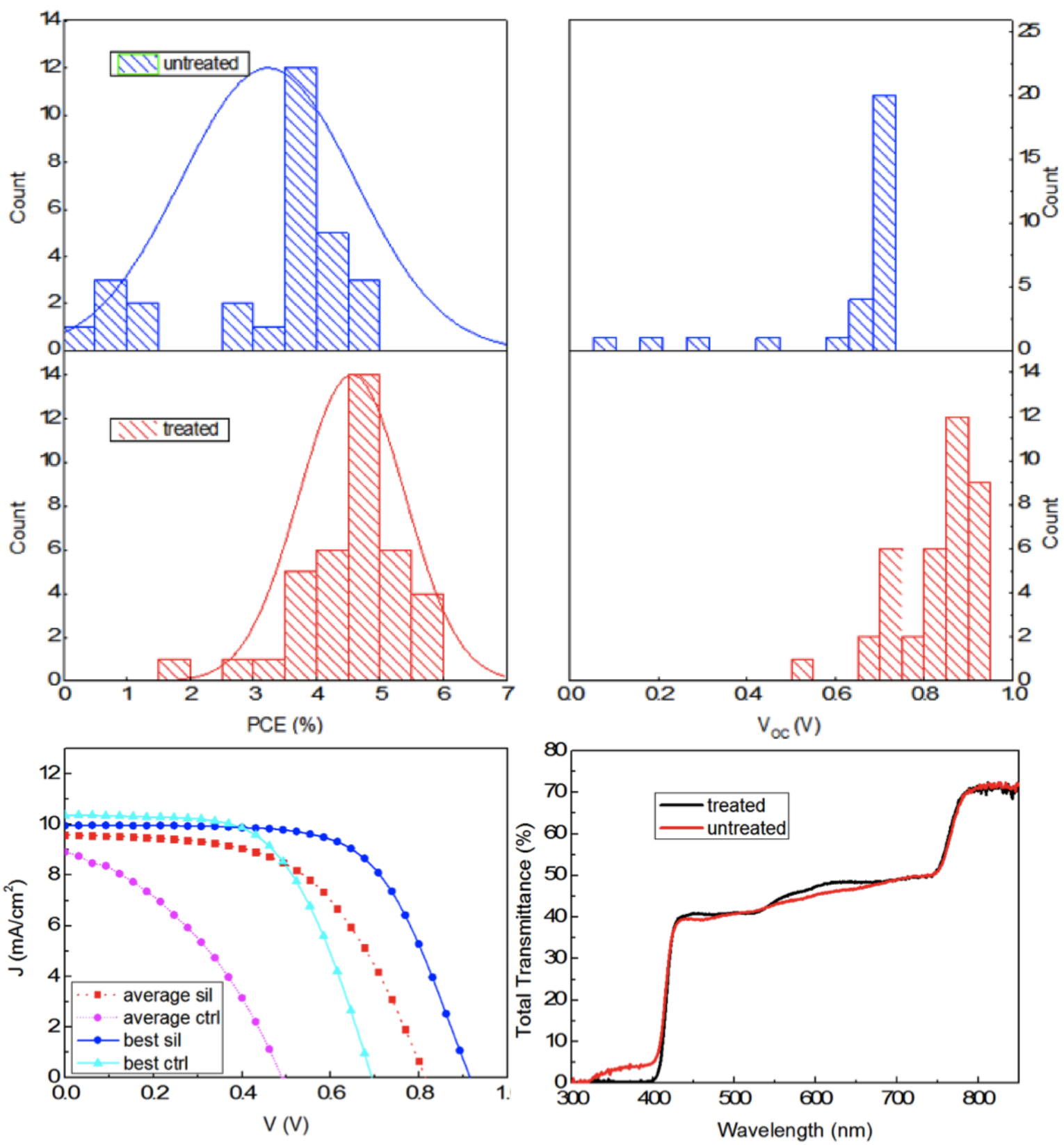

Figure 2. Histograms and fitted normal distributions of the measured $\mathrm{V}_{\mathrm{OC}}$ of semitransparent dewet perovskite cells before (a) and after (b) OTS treatment. Histograms and fitted normal distributions of the measured PCE of semitransparent dewet perovskite cells before (c) and after (d) OTS treatment. Averaged JV-curves of 15 semitransparent perovskite solar cell devices with non-transparent Au top-electrode before (magenta) and after (red) OTS treatment (including all devices above $0.1 \% \mathrm{PCE}$ ). JV-curves (scan speed: $0.38 \mathrm{~V} / \mathrm{s}$, scan direction: forward bias to short-circuit) of best preforming semitransparent perovskite solar cell devices with non-transparent Au top-electrode before (cyan) and after (blue) OTS treatment (e). Total 


\section{WILEY-VCH}

transmittance UV-vis spectra of top-electrode less semitransparent before (red) and after (blue) OTS treatment (f).
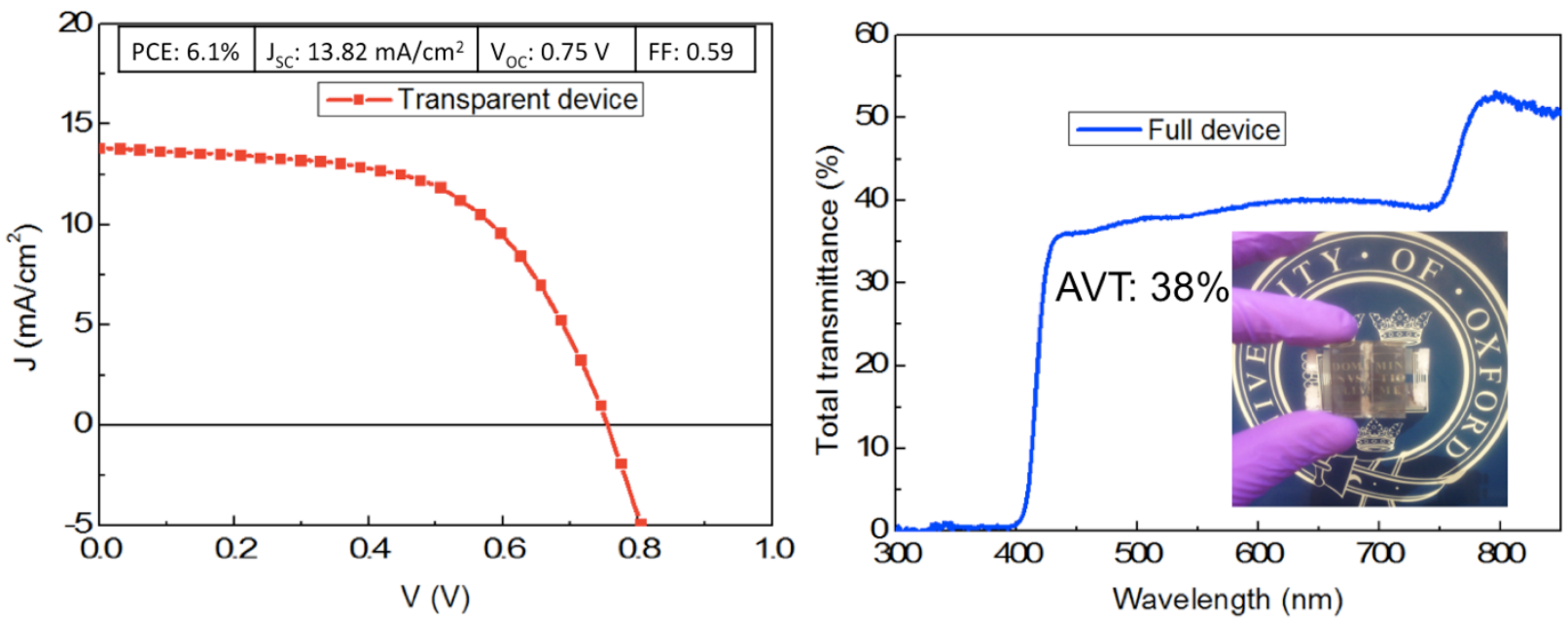

Figure 3. Characteristic JV-curve (scan speed: $0.38 \mathrm{~V} / \mathrm{s}$, scan direction: forward bias to shortcircuit) of best performing semitransparent dewet perovskite solar cell after OTS treatment with transparent top-electrode (a). Total transmittance UV-vis spectrum of best performing semitransparent perovskite cell after OTS treatment with transparent top-electrode (b). Photograph of best performing neutral colour semitransparent device with transparent topelectrode.

Table 1. Average and maximum performance parameters of OTS treated $(n=39)$ and untreated $(\mathrm{n}=28)$ semitransparent perovskite devices.

\begin{tabular}{lcccc}
\hline Device type & $\begin{array}{c}\mathrm{J}_{\mathrm{sc}} \\
{[\mathrm{mA} \mathrm{cm}-2]}\end{array}$ & $\begin{array}{c}\mathrm{V}_{\mathrm{oc}} \\
{[\mathrm{V}]}\end{array}$ & $\begin{array}{c}\mathrm{FF} \\
{[-]}\end{array}$ & $\begin{array}{c}\text { PCE } \\
{[\%]}\end{array}$ \\
\hline OTS treated avg. & $9.6 \pm 0.8$ & $0.83 \pm 0.09$ & $0.56 \pm 0.06$ & $4.6 \pm 0.8$ \\
\hline Control avg. & $8.9 \pm 1.4$ & $0.60 \pm 0.16$ & $0.52 \pm 0.13$ & $3.2 \pm 1.4$ \\
\hline OTS treated max & 10.0 & 0.92 & 0.64 & 5.9 \\
\hline Control max & 10.4 & 0.69 & 0.60 & 4.3
\end{tabular}




\section{WILEY-VCH}

We utilize silane based, electrically insulated molecular layers that attach preferentially in the pin-hole forming regions without hindering the charge transport in the active areas. Using a $\mathrm{Ni}$ microgrid based transparent top electrode, we demonstrate $6.1 \%$ PCE with $38 \%$ average visible transparency for a neutral colour semitransparent perovskite solar cell.

perovskite, solar cell, semi-transparent, siloxane, shunt-blocking

M. T. Hörantner ${ }^{\dagger}$, P. K. Nayak ${ }^{\dagger}$, S. Mukhopadhyay, K. Wojciechowski, C. Beck, D. McMeekin, B. Kamino, G. E. Eperon and H. J. Snaith*

\section{Shunt-Blocking Layers for Semitransparent Perovskite Solar Cells}

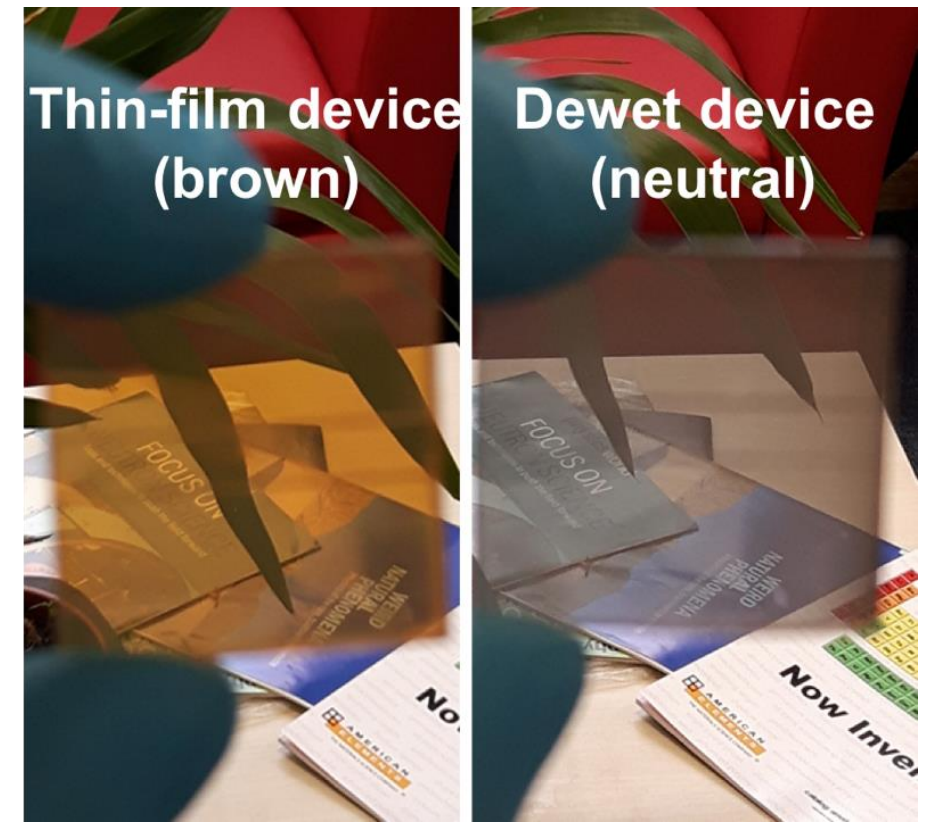

